\title{
Clinical and pathological factors predictive of response to neoadjuvant chemotherapy in breast cancer: A single center experience
}

\author{
SALVATORE DEL PRETE ${ }^{1}$, MICHELE CARAGLIA ${ }^{2}$, AMALIA LUCE $^{2}$, LILIANA MONTELLA ${ }^{1}$, \\ GENNARO GALIZIA $^{3}$, PASQUALE SPERLONGANO ${ }^{3}$, GREGORIO CENNAMO ${ }^{1}$, EVA LIETO ${ }^{3}$, \\ ELENA CAPASSO $^{1}$, OLGA FIORENTINO ${ }^{4}$, MARIA ALIBERTI ${ }^{1}$, ANNAMARIA AURICCHIO ${ }^{3}$, \\ PATRIZIA IODICE $^{1}$ and RAFFAELE ADDEO ${ }^{1}$

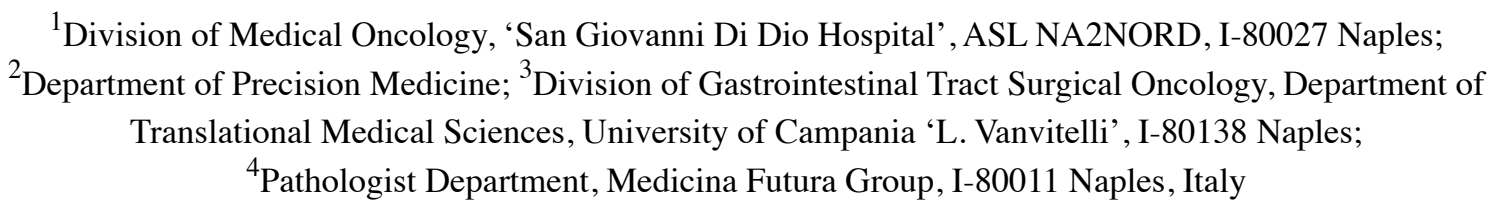

Received February 3, 2019; Accepted May 9, 2019

DOI: $10.3892 / 01.2019 .10729$

\begin{abstract}
Neoadjuvant chemotherapy (NAC) of breast cancer (BC) improves outcomes, especially in patients with locally advanced and inflammatory cancer. Further insight into clinic-pathological factors influencing outcomes is essential to define the optimal therapeutic strategy for each category of patients and to predict the response to the treatment. In total, 117 patients with $\mathrm{BC}$ were treated with NAC with or without trastuzumab between 2010 and 2015. The histologic response to NAC was defined as a pathological complete response (pCR) when there was no evidence of residual invasive tumor in the breast or axillary lymph nodes. Relapse-free survival (RFS) was estimated using the Kaplan-Meier method and compared using log rank analysis. P-value $<0.05$ was considered statistically significant. The median age of the 117 patients enrolled in the present study was 52 years (age range, $35-85$ years). The overall response rate (complete and partial responses) assessed by radiological and pathological evaluation were 76 and $72 \%$, respectively. pCR was achieved in 35 out of 117 patients $(\sim 30 \%)$. In total, 6 patients (5\%) developed progressive disease during chemotherapy. The RFS was 85 months ( $\mathrm{SE}=3$; 95\% CI 79-91). The median was not reached and the mean follow-up time was 55 months (median 52 months; range 11-100 months). In this time, 20 patients (17\%) experienced tumor recurrence. From the univariate analysis, the pathological response was significantly associated with receptor-based subtype,
\end{abstract}

Correspondence to: Professor Michele Caraglia, Department of Precision Medicine, University of Campania 'L. Vanvitelli', Via L. De Crecchio 7, I-80138 Naples, Italy

E-mail: michele.caraglia@unicampania.it

Key words: breast cancer, neoadjuvant chemotherapy, triple negative, estrogen receptor, progesterone receptor menopausal status and T-stage. From the multivariate analysis by using linear multiple regression and including receptormenopausal status and T-stage, the model was not significant $(\mathrm{P}=0.062)$. However, by using the multiple logistic regression, and including age, $\mathrm{pCR}$ was significantly associated with ER+ HER2neg $(\mathrm{P}=0.006)$, T2 $(\mathrm{P}=0.043)$ and T3 $(\mathrm{P}=0.018)$. T-stage, menopausal status and receptor status are significantly associated with the pathological response in patients with inoperable BC treated with NAC.

\section{Introduction}

Preoperative neoadjuvant chemotherapy (NAC) is established as a therapeutic avenue for selected high-risk BCs, with tumors $\geq 2 \mathrm{~cm}$ and for locally advanced (including initially ineligible for resection) disease (1). Breast cancer is divided into three groups based on the expression or not of different markers such as estrogen receptor (ER), progesterone receptor $(\mathrm{PgR})$ and human epidermal growth factor receptor 2 (HER2): Hormone receptor positive/ HER2 negative, HER2 positive, and triple-negative that does not have any of the three molecular markers. The therapeutic intervention consists of endocrine therapy for all Hormone Receptor (HR) positive tumors with some patients requiring chemotherapy (adriamycin, cyclophosphamide, paclitaxel or docetaxel); chemotherapy plus HER2-targeted therapy for HER 2 positive and chemotherapy alone for triple-negative breast cancer (2). The use of NAC in BC treated with surgery is currently increasing because of the chance of measuring early in-vivo response to systemic treatment and to achieve higher rates of breast conserving surgery (3). Neoadjuvant treatment modalities require a close collaboration between oncologists, surgeons, radiologists and pathologists to attain the best results.

The most important parameter for successful treatment and improvement in overall survival (OS) is the achievement of a pCR although the identification of clinical and 
pathological parameters predictive of treatment response and best survival outcomes remains not still clearly defined. Preliminary identification of patient subgroups with high $\mathrm{pCR}$ rates could preferably refer them to neoadjuvant treatment strategies. Despite extensive clinical investigations, it has not yet been clarified whether NAC would result in improved survival in comparison with the standard adjuvant setting in any subgroups of patients with BC (4).

However, comparable data between adjuvant and neoadjuvant settings $(5,6)$ have changed the $\mathrm{BC}$ treatment, with an increased use of NAC. These data have stimulated the formation of an international expert panel consensus to provide recommendations on the use of neoadjuvant therapy. In short, the panel suggests that NAC should be considered in any individual patient for whom adjuvant chemotherapy is indicated (7).

$\mathrm{BC}$ is increasingly recognized as a heterogeneous disease showing a different response to treatment according to defined clinical and pathological parameters. Additionally, changes in some pathological parameters during each $\mathrm{BC}$ history require new tumor biopsy and even complicate the $\mathrm{BC}$ treatment. The prognostic value of these changes is also not well defined $(8,9)$. Chemotherapy is especially effective in the treatment of endocrine insensitive tumors, and such therapeutic benefit can be assumed for patients with triple-negative, or HR negative and HER 2 positive BC $(10,11)$. In case of HER 2 positive tumors, an anti-HER 2 agent can be administered as part of the preoperative treatment, and according to preliminary clinical data, dual HER2 blockade can offer an additional therapeutic value (12). However, dose escalation, kind of chemotherapy regimen and number of cycles are still debating matters.

In this study, we reported retrospective data concerning 117 BC patients treated with NAC in a single institution. All available clinic-pathological parameters were analyzed to evaluate the correlation, if any, with response and survival outcomes.

\section{Patients and methods}

Patient selection. Data from 117 patients with BC diagnosed by needle aspiration cytology and histopathology biopsy and treated from March 2010 to December 2015 with various regimens of NAC at a single center were reviewed. All patients provided informed consent and Local Ethical Committee of San Giovanni di Dio Hospital of Frattamaggiore gave formal approval to this retrospective study (approval no. 1250 on 20th February 2018). Eligible patients were women aged $\geq 18$ years with localized primary BC suitable for primary medical treatment with or without regional lymph node metastases, with adequate bone marrow, renal, hepatic, and cardiac functions, no other uncontrolled medical or psychiatric disorders and with an ECOG performance status of 0-1. The main exclusion criteria were distant metastases, other malignancy in the past two years (except for radically treated basal or squamous cell carcinoma of the skin or carcinoma in situ of the cervix), and pregnancy or lactation.

Clinical assessment. At baseline all patients underwent clinical assessment, hematology and chemistry and core needle biopsies that were performed either free-hand or under ultra-sound guidance. For each patient we recorded baseline tumor size by ultrasound and mammography, nodal status involvement by ultrasound-guided fine-needle aspiration or, when negative, by sentinel node biopsy, type of NAC and type of surgery (S). pCR defined as the absence of invasive cells in the breast and the lymph nodes regardless of Ductal Carcinoma In Situ (DCIS). Immunohistochemistry (IHC) subtypes were defined according to Estrogen (ER) and progesterone receptor (PgR), Ki-67 level (nuclear antigen expressed in cycling cells), and HER2 status.

Clinical and radiological response evaluations were performed after two, four and six courses of treatment. All patients underwent Positron Emission Tomography (PET) at baseline and before surgery.

Tumors were staged according to Tumor Node Metastasis (TNM) staging system (7th edition). ER and PgR status were determined by IHC and was considered positive if tumor cells were $\geq 1 \%$. Tumors with a score of $3+$ (strong homogeneous staining) were considered HER2-positive. In case of 2+ scores (moderate homogeneous staining) chromogenic in situ hybridization (CISH) was used to determine amplification. The tumor margins were defined as a clear pathologic margin if the distance was $>2 \mathrm{~mm}$ by microscopy evaluation. The clinic-pathological data at baseline are summarized in Table I. The clinical pathological protocols used for the collection of the data were recorded at the Unit of Oncology of San Giovanni di Dio Hospital in Frattamaggiore.

Therapeutic management of patients. Neoadjuvant regimens of chemotherapy were summarized in Table II. Number of chemotherapy cycles administered was guided by tumor evaluation and ranged between 4 and at most 6 cycles. Recommendation for surgery was made after evaluation of a team of $\mathrm{BC}$ specialists including radiologists, medical oncologists and surgeons. Treatment decisions were mainly based on patient's desires and surgical considerations involving the breast-tumor index, age, multifocality, localization and response were obtained after NAC. All patients with proven axillary lymph node metastases prior to NAC underwent to an axillary lymph node dissection (ALND) at levels I and II with level III sampling after NAC. Level III sampling was done to stage for adjuvant radiotherapy indications. Patients undergoing to breast-conserving surgery received radiation to the breast with a boost to the tumor bed. The indication for loco-regional radiation therapy (chest wall and regional nodal basins) was based on the original staging. Hormone receptor-positive patients received adjuvant endocrine treatment for at least 5 years and HER2-positive patients were treated with trastuzumab for 1 year.

Statistical anlaysis. RFS was estimated by the Kaplan-Meier method and compared using the log rank analysis. P-values were considered statistically significant when $<0.05$. The statistical analyses were performed using the chi-square test and conducted by SPSS software (version 17.0).

\section{Results}

Patient characteristics. The median age of the patients was 52.8 years (range $35-85$ years). Fifty-three (45\%) were pre- and $64(55 \%)$ were post-menopausal women. Most patients had ductal invasive carcinoma (approximately 90\%). T2 and T4 
Table I. Patient characteristics.

\begin{tabular}{|c|c|}
\hline Characteristic & Result \\
\hline \multicolumn{2}{|l|}{ Age } \\
\hline Age median (years) & 52.8 \\
\hline Age range (years) & $35-85$ \\
\hline \multicolumn{2}{|l|}{ Menstrual status } \\
\hline Premenopausal & 53 \\
\hline Postmenopausal & 64 \\
\hline \multicolumn{2}{|l|}{ Histology } \\
\hline Ductal & 105 \\
\hline Lobular & 7 \\
\hline Mixed & 5 \\
\hline \multicolumn{2}{|l|}{ T-stage } \\
\hline $\mathrm{T} 1$ & 12 \\
\hline $\mathrm{T} 2$ & 49 \\
\hline $\mathrm{T} 3$ & 15 \\
\hline $\mathrm{T} 4$ & 42 \\
\hline \multicolumn{2}{|l|}{ N-stage } \\
\hline No & 18 \\
\hline $\mathrm{N} 1$ & 26 \\
\hline $\mathrm{N} 2 / \mathrm{N} 3$ & 66 \\
\hline $\mathrm{NX}$ & 7 \\
\hline \multicolumn{2}{|l|}{ Receptor-based subtype } \\
\hline ER-positive (ER+; HER2-) & 67 \\
\hline Triple-negative (ER-; PR-; HER2-) & 15 \\
\hline HER2+ & 35 \\
\hline \multicolumn{2}{|l|}{ Ki67 status } \\
\hline Low $(<14 \%)$ & 24 \\
\hline Intermediate $(14-30 \%)$ & 52 \\
\hline High $(>30 \%)$ & 41 \\
\hline
\end{tabular}

ER, estrogen receptor; HER2, human epidermal growth factor receptor; PR, progesterone receptor; Ki67, proliferation marker protein Ki-67.

tumors were the most represented in our series: 42 and $36 \%$, respectively. Lymph node involvement classified as N2/N3 was present in $56 \%$ of the patients. Hormone positive, HER 2 negative tumors were $57 \%$ of the patients. HER2 positive tumors were about $30 \%$ of the patients. Intermediate-high proliferating tumors, expressed by Ki-67, were about 79\% (Table I).

Effects of NAC regimen. Chemotherapy used for NAC was predominantly an anthracycline-based regimen (67\% of the patients) as shown in Table II. A HER 2 targeted therapy was used in $16 \%$ of the patients. In $19 \%$ of the patients a liposomal doxorubicin was used. In $14 \%$ of the patients, belonging to the last enrolled patients, the novel treatment with double antibodies (pertuzumab and trastuzumab) plus docetaxel was used (Table II). The number of patients treated with trastuzumab was low because the combination of trastuzumab with anthracyclines could induce sub-clinical or clinical cardiac failure.
Table II. pCR correlation with neoadjuvant treatment.

\begin{tabular}{lccc}
\hline $\begin{array}{l}\text { Chemotherapy } \\
\text { regimen }\end{array}$ & $\begin{array}{c}\text { Patient } \\
\text { number }\end{array}$ & $\begin{array}{c}\text { pCR } \\
\text { number }\end{array}$ & $\begin{array}{c}\text { pCR } \\
\text { rate }(\%)\end{array}$ \\
\hline $\mathrm{EC} \rightarrow$ TXT & 5 & 0 & 0 \\
$\mathrm{EC} \rightarrow$ TXT + H & 2 & 0 & 0 \\
$\mathrm{TMC}$ & 16 & 1 & 6 \\
$\mathrm{TEC}+\mathrm{H}$ & 11 & 2 & 18 \\
$\mathrm{TEC}$ & 60 & 17 & 28 \\
$\mathrm{TMC}+\mathrm{H}$ & 6 & 2 & 33 \\
PERT/H/TXT & 17 & 13 & 76 \\
\hline
\end{tabular}

EC, Epirubicin + Cyclophosphamide; TXT, Taxotere; H, Herceptin (Trastuzumab); TMC, Docetaxel + Myocet (liposomal doxorubicin) + Cyclophosphamide; TEC, Docetaxel + Epirubicin + Cyclophosphamide; PERT, Pertuzumab.

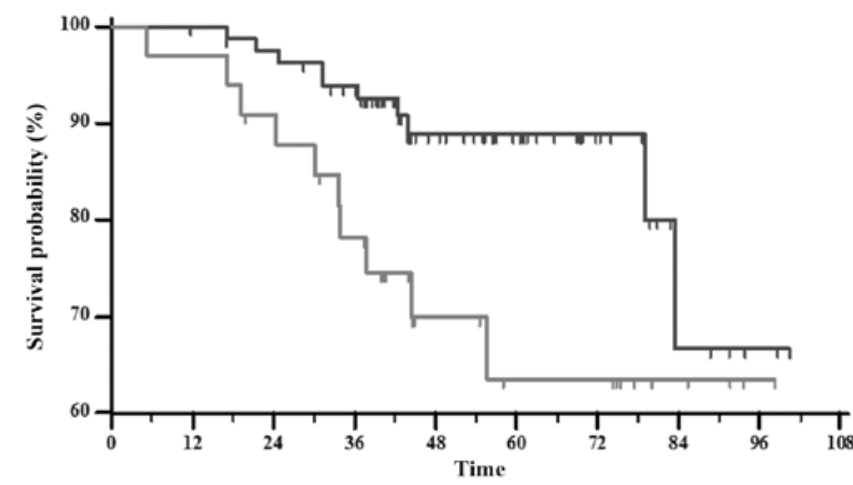

Figure 1. Kaplan-Meier curve of relapse-free survival estimated by dividing patients into two categories and compared using log rank test, $\mathrm{P}=0.0210$. Patients with complete and partial response (dark grey) compared with the stable and progressive disease group (light grey).

All patients had a positive PET scan at the tumor site. About $73 \%$ of the patients showed significant uptake at the level of the axilla. Overall Response Rate (ORR) was 76 and $72 \%$ when assessed by radiological and pathological evaluation, respectively. In 40 out of 117 patients (34\%) a conservative treatment was allowed. In details, we recorded $46 \mathrm{CRs}$ and 43 partial responses (PRs) by radiology (Table III). Thirty-five pCRs (pCR rate about 30\%) and 49 pPRs were documented by pathology (Table III). Among the 15 patients with triple-negative tumors, a conservative surgical treatment was performed in 6 patients and in 6 instances a pathological response was obtained. Three triple negative patients relapsed during follow-up: One patient at lymph nodes, one at lymph nodes and bone and one at bone and visceral sites.

Statistical correlation between pathological responses, and clinical and pathological features. Comparing all together complete and partial pathologically responders to the patients with SD and PD, the RFS difference was highly statistically significant $(\mathrm{P}=0.0210)$ as shown in Fig. 1. However, pCR does not significantly correlate with either 
Table III. Response rates by method of assessment.

\begin{tabular}{lcccccc}
\hline Clinical assessment & Total number & ORR $(\%)$ & CR number & PR number & SD number & PD number \\
\hline Radiological & 117 & 76 & 46 & 43 & 23 & 5 \\
Pathological & 117 & 72 & 35 & 49 & 28 & 5 \\
\hline
\end{tabular}

ORR, overall response rate; $\mathrm{CR}$, complete response; $\mathrm{PR}$, partial response; $\mathrm{SD}$, stable disease; $\mathrm{PD}$, progressive disease.
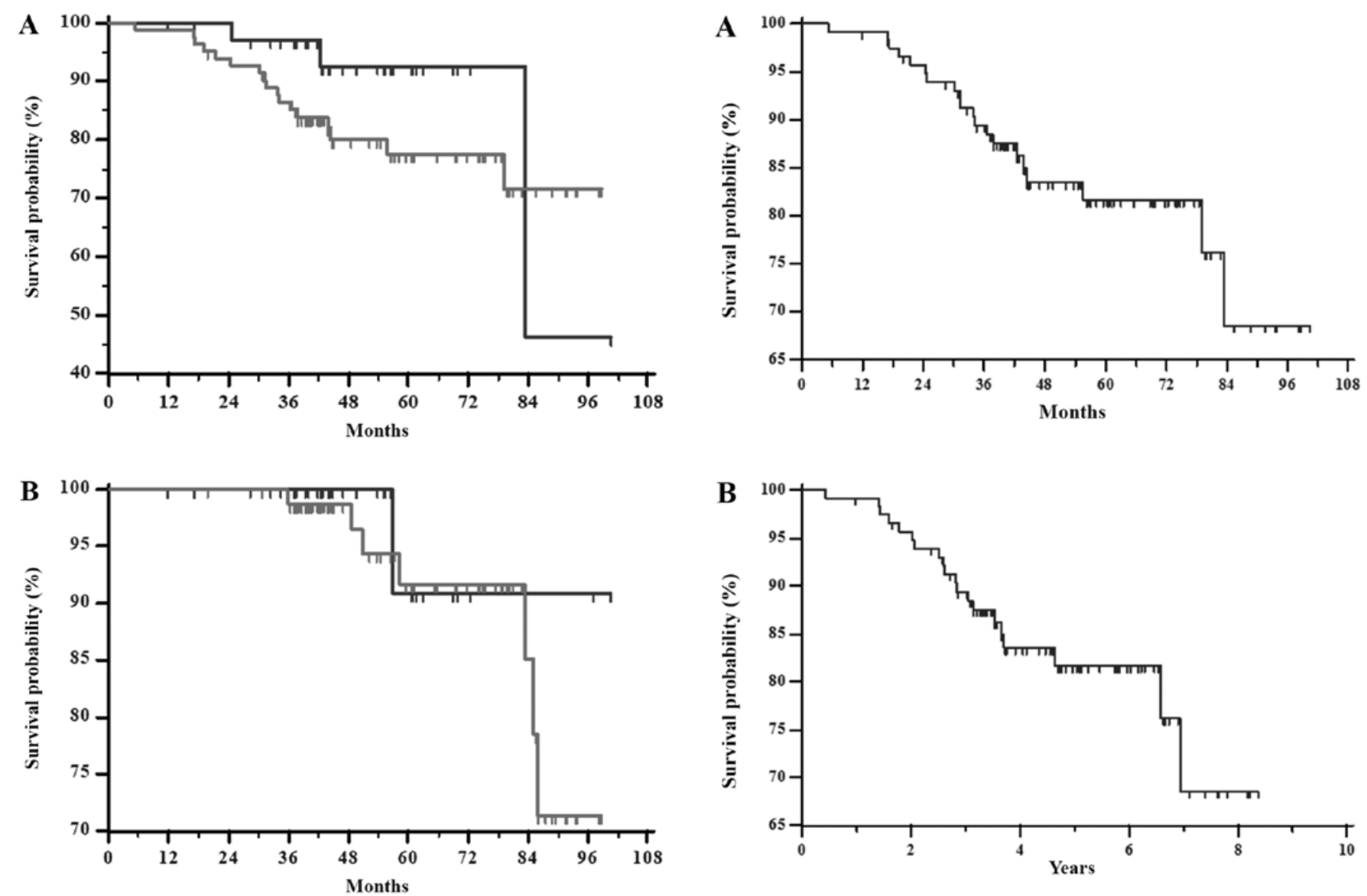

Figure 2. Kaplan-Meier curve estimated by dividing patients into two categories. Patients with a complete pathological response (dark grey) compared with the overall population, $\mathrm{PR}+\mathrm{SD}+\mathrm{PD}$ (light grey). (A) Relapse-free survival is presented, $\mathrm{P}=0.1852$. (B) Overall survival is presented, $\mathrm{P}=0.5599$. $\mathrm{PR}$, partial response; $\mathrm{SD}$, stable disease; $\mathrm{PD}$, progressive disease.

RFS or overall survival (OS) $(\mathrm{P}=0.1852$ and $\mathrm{P}=0.5599$, respectively) (Fig. $2 \mathrm{~A}$ and $\mathrm{B}$, respectively), when compared to patients with pPRs + pSD + PD. There was no significant difference between clinical response and Ki-67 labeling index $(\mathrm{P}=0.9705)$. Likewise, the statistical analysis showed no differences between pathological responses and Ki-67 labeling index $(\mathrm{P}=0.2867)$ (Table IV).

By chi-square test the combination of an anthracycline-based regimen plus an anti-HER 2 therapy was significantly associated with $\mathrm{pCR}(\mathrm{P}=0.00025712)$.

The RFS mean for all groups (117 patients) was 85 months $(\mathrm{SE}=3 ;$ 95\% CI 79-91). The median was not reached and the mean follow-up time was 55 months (median 52 months; range 11-100 months). In this time, about twenty patients

Figure 3. Kaplan-Meier curve of RFS estimated for all patients using log rank test, $\mathrm{P}<0.001$. (A) RFS mean for all group (117 patients) was 85 months ( $\mathrm{SE}=3$; $95 \%$ CI 79-91). The median was not reached; the mean follow-up time was 55 months (median 52 months; range 11-100 months). In this time, twenty patients (17\%) experienced tumor recurrence. (B) 1-7 year RFS rates were 99.1, $95.7,89.4,83.5,81.7,81.7$ and $68.6 \%$, respectively. RFS, relapse-free survival.

(17\%) experienced tumor recurrence (Fig. 3A). The 1-7 year RFS rates were 99.1, 95.7, 89.4, 83.5, 81.7, 81.7 and $68.6 \%$, respectively (Fig. 3B).

At univariate analysis, pathological response was significantly associated with receptor-based subtype $(\mathrm{P}=0.0001)$, menopausal status $(\mathrm{P}=0.0368)$ and $\mathrm{T}$-stage $(\mathrm{P}=0.0007)$. On the contrary, obesity $(\mathrm{P}=0.6128)$, histological subtype $(\mathrm{P}=0.3238)$, Ki67 status $(\mathrm{P}=0.1747), \mathrm{N}$-stage $(\mathrm{P}=0.8914)$ and age $(\mathrm{P}=0.0730)$ did not seem to correlate with pathological response in this patients' series (Table $\mathrm{V}$ ).

At multivariate analysis by using linear multiple regression and including receptor status, menopausal status and T-stage, the model was not significant for menopausal status $(\mathrm{P}=0.062)$. 
Table IV. Clinical and pathological response after neoadjuvant chemotherapy by Ki67 labeling index.

Ki67 labeling index

\begin{tabular}{lcccc}
\cline { 2 - 4 } Therapy response & Low & Intermediate & High & P-value \\
\hline Clinical response & 17 & & 30 & 0.9705 \\
PR + CR & 7 & 15 & 11 & \\
SD + PD & 6 & 13 & 16 & 0.2867 \\
Pathological response & 18 & 39 & 25
\end{tabular}

pCR, pathological complete response; CR, complete response; PR, partial response; $\mathrm{SD}$, stable disease; PD, progressive disease; Ki67, proliferation marker protein Ki-67.

Table V. Correlation between pathological responses and clinical and pathological features.

\begin{tabular}{|c|c|c|c|}
\hline Clinical and pathological features & pCR breast and axilla number & Not-pCR & P-value \\
\hline \multicolumn{4}{|l|}{ Histological subtype } \\
\hline Ductal & 33 & 72 & \multirow{3}{*}{0.3238} \\
\hline Lobular & 2 & 5 & \\
\hline Mixed & 0 & 5 & \\
\hline \multicolumn{4}{|l|}{ Receptor-based subtype } \\
\hline ER-positive (ER+; HER2-) & 10 & 59 & \multirow[t]{3}{*}{0.0001} \\
\hline Triple-negative (ER-; PR-; HER2-) & 7 & 8 & \\
\hline HER2+ & 18 & 15 & \\
\hline \multicolumn{4}{|l|}{ Ki67 status } \\
\hline Low $(<14 \%)$ & 6 & 18 & \multirow{3}{*}{0.1747} \\
\hline Intermediate $(14-30 \%)$ & 13 & 39 & \\
\hline High $(>30 \%)$ & 16 & 25 & \\
\hline \multicolumn{4}{|l|}{ Menopausal status } \\
\hline Premenopausal & 21 & 32 & \multirow[t]{2}{*}{0.0368} \\
\hline Postmenopausal & 14 & 50 & \\
\hline \multicolumn{4}{|l|}{ T-stage } \\
\hline $\mathrm{T} 1$ & 8 & 3 & \multirow{4}{*}{0.0007} \\
\hline $\mathrm{T} 2$ & 10 & 39 & \\
\hline $\mathrm{T} 3$ & 1 & 14 & \\
\hline $\mathrm{T} 4$ & 16 & 26 & \\
\hline \multicolumn{4}{|l|}{$\mathrm{N}$-stage } \\
\hline N0 & 4 & 14 & \multirow{4}{*}{0.8914} \\
\hline N1 & 8 & 18 & \\
\hline $\mathrm{N} 2 / \mathrm{N} 3$ & 20 & 44 & \\
\hline NX & 3 & 6 & \\
\hline \multicolumn{4}{|l|}{ BMI } \\
\hline Overweight & 15 & 41 & \multirow[t]{2}{*}{0.6128} \\
\hline Normal & 20 & 41 & \\
\hline \multicolumn{4}{|l|}{ Age } \\
\hline$>50$ & 14 & 48 & \multirow[t]{2}{*}{0.0730} \\
\hline$<50$ & 21 & 34 & \\
\hline
\end{tabular}

pCR, pathological complete response; ER, estrogen receptor; HER2, human epidermal growth factor receptor; PR, progesterone receptor; Ki67, proliferation marker protein Ki-67; BMI, body mass index. 
However, by using the multiple logistic regression, and including age, $\mathrm{pCR}$ was significantly associated with ER+ HER2neg ( $\mathrm{P}=0.006), \mathrm{T} 2(\mathrm{P}=0.043)$, and T3 $(\mathrm{P}=0.018)$.

Histological heterogeneity of breast cancer. Fourteen tumor histotypes changed: 5 mixed ductal/lobular tumors became ductal (4 cases) and lobular (1 case), 8 ductal carcinomas became lobular ( 7 cases) and ductal hyperplasia (1 case), one lobular carcinoma became ductal. ER/PgR and HER2 status changed in 15 out of 117 patients (12\%). In particular, 5 patients became HER 2 positive and 5 patients became negative (respectively about $4 \%$ ). ER/PgR status changed in 7 patients $(6 \%)$ with 5 patients becoming hormone-responsive.

\section{Discussion}

In this retrospective review of clinical practice treatment with NAC of BC patients, we obtained a high radiological and pathological response rate. The association of an anthracycline-based regimen plus an anti-HER2 therapy was significantly associated with high $\mathrm{pCR}$ rate. Moreover, clinical and pathological response was independent from proliferation rate as expressed by Ki-67 status, reflecting an association to still unspecified molecular features more than to the simple proliferation rate of the neoplasm as previously reported. In this series, pCR does not significantly correlate with both RFS and OS, differently from most published studies. A meta-analysis of randomized clinical trials for resectable BC comparing the survival benefit of NAC vs. postoperative chemotherapy showed that NAC cycles, the total number of chemotherapy cycles, administration of tamoxifen, administration of adjuvant chemotherapy, or type of NAC regimen, did not influence OS. The pooled HR estimate for RFS was influenced by anthracycline-containing regimens. Patients with a pCR had superior survival outcomes compared with patients who had residual disease (5). However, we found a significant correlation between the pathological response and the RFS when compared to stable or progressive disease. In the present study, an anthracycline-based regimen plus the anti-HER2 agent trastuzumab showed better results in term of pCR. Interestingly, when treating HER2 positive tumours the best response is given by trastuzumab and pertuzumab combined with docetaxel. The responses observed in this subset of patients is online with previously published trials $(13,14)$. Moreover, the synergism between pertuzumab and trastuzumab is not surprising if we consider that this kind of tumours are likely driven by an overexpression and hyperactivation of peptide growth factor receptors and, therefore, are more likely responsive to the block of these pathways. HER2-positive breast cancers are more aggressive than other types of $\mathrm{BC}$ and anti-HER 2 agents bind different domains of HER2 receptor inducing a dual blockade of the receptor and consequent inhibition of the downstream signaling processes associated with tumor growth and progression. The concomitant use of the two antibodies can have synergistic effects based upon the different biological effects triggered by the two different weapons: Trastuzumab activates immunological effects (i.e.: ADCC) and blocks receptor activation and pertuzumab inhibits receptor dimerization thus specifically blocking the different signal transduction activated by the receptors overcoming resistance to trastuzumab due to HER2 truncations (15). However, it is still not clear and defined how to predict the response of BC to NAC regimens and how to select the best combination in patients. Molecular research on genetic characteristics of $\mathrm{BC}$ could be useful in the future to predict the best choice in this subset of patients as already experienced some years ago for taxanes (16). In the present study we recorded also a change in some cases of the pathological molecular features of $\mathrm{BC}$ after the treatment of the patients with NAC. In fact, HER2 status changed in $8 \%$ of cases. $\mathrm{ER} / \mathrm{PgR}$ status changed in $6 \%$ of the patients. These data are similar to previously reported similar series (17). The difference between IHC from preliminary biopsy and definitive surgical specimens may reflect not only different kind of samples but also a change in tumor itself conditioned by NAC.

Obesity does not seem to correlate with pathological response in this patients' series. Previous studies assessed the value of obesity in patients treated with NAC. Obesity seems to have a negative impact on survival that is independent from chemotherapy dosing (18-21).

Although the reduced number of patients and the retrospective analysis limited the value of this study, the data reported reflect the clinical practice of a single institution and suggest evaluating T-stage, menopausal status and receptor status in patients with inoperable $\mathrm{BC}$ treated with NAC to predict the pathological response. Despite the increased knowledge of distinctive clinical and pathological parameters and insights into genetic variability of $\mathrm{BC}$, a standardized model predictive of response to NAC is not presently available. A tailored evaluation of presumptive better chemotherapeutic regimens for each tumor subtype is desirable in the next future.

\section{Acknowledgements}

Not applicable.

\section{Funding}

This article was supported by a grant from POR CAMPANIA FESR 2014/2020

\section{Availability of data and materials}

All data generated and/or analyzed during the present study are included in this published article.

\section{Authors' contributions}

SDP, MC, AL, LM, PS, GC, EC, OF, MA, PI and RA designed the study and collected the data. RA and MC wrote the paper. GG, EL and AA performed the statistical analysis.

\section{Ethics approval and consent to participate}

All patients provided informed consent and Local Ethical Committee of San Giovanni di Dio Hospital of Frattamaggiore gave formal approval to this retrospective study (approval no. 1250 on 20th February 2018).

\section{Patient consent for publication}

Not applicable. 


\section{Competing interests}

The authors declare that they have no competing interests.

\section{References}

1. Thompson AM and Moulder-Thompson SL: Neoadjuvant treatment of breast cancer. Ann Oncol 23 (Suppl 10): x231-x236, 2012

2. Waks AG and Winer EP: Breast cancer treatment: A review. JAMA 321: 288-300, 2019.

3. Untch M, Konecny GE, Paepke S and von Minckwitz G: Current and future role of neoadjuvant therapy for breast cancer. Breast 23: 526-537, 2014.

4. Rubovszky G and Horváth Z: Recent advances in the neoadjuvant treatment of breast cancer. J Breast Cancer 20: 119-131, 2017

5. Chen Y, Shi XE, Tian JH, Yang XJ, Wang YF and Yang KH: Survival benefit of neoadjuvant chemotherapy for resectable breast cancer: A meta-analysis. Medicine (Baltimore) 97: e10634, 2018.

6. Early Breast Cancer Trialists' Collaborative Group (EBCTCG): Long-term outcomes for neoadjuvant versus adjuvant chemotherapy in early breast cancer: Meta-analysis of individual patient data from ten randomised trials. Lancet Oncol 19: 27-39, 2018.

7. Kaufmann M, Hortobagyi GN, Goldhirsch A, Scholl S, Makris A, Valagussa P, Blohmer JU, Eiermann W, Jackesz R, Jonat W, et al: Recommendations from an international expert panel on the use of neoadjuvant (primary) systemic treatment of operable breast cancer: An update. J Clin Oncol 24: 1940-1949, 2006.

8. Yang L, Zhong X, Pu T, Qiu Y, Ye F and Bu H: Clinical significance and prognostic value of receptor conversion in hormone receptor positive breast cancers after neoadjuvant chemotherapy. World J Surg Oncol 16: 51, 2018

9. Yoshida A, Hayashi N, Suzuki K, Takimoto M, Nakamura S and Yamauchi H: Change in HER2 status after neoadjuvant chemotherapy and the prognostic impact in patients with primary breast cancer. J Surg Oncol 116: 1021-1028, 2017.

10. Straver ME, Rutgers EJ, Rodenhuis S, Linn SC, Loo CE, Wesseling J, Russell NS, Oldenburg HS, Antonini N and Vrancken Peeters MT: The relevance of breast cancer subtypes in the outcome of neoadjuvant chemotherapy. Ann Surg Oncol 17: 2411-2418, 2010

11. Curigliano G, Burstein HJ, P Winer E, Gnant M, Dubsky P, Loibl S, Colleoni M, Regan MM, Piccart-Gebhart M, Senn HJ, et al: De-escalating and escalating treatments for early-stage breast cancer: The St. Gallen International expert consensus conference on the primary therapy of early breast cancer 2017. Ann Oncol 28: 1700-1712, 2017.
12. Debiasi M, Polanczyk CA, Ziegelmann P, Barrios C, Cao H, Dignam JJ, Goss P, Bychkovsky B, Finkelstein DM, Guindalini RS, et al: Efficacy of anti-HER2 agents in combination with adjuvant or neoadjuvant chemotherapy for early and locally advanced HER2-positive breast cancer patients: A network meta-analysis. Front Oncol 8: 156, 2018.

13. Swain SM, Ewer MS, Viale G, Delaloge S, Ferrero JM, Verrill M, Colomer R, Vieira C, Werner TL, Douthwaite H, et al: Pertuzumab, trastuzumab, and standard anthracycline- and taxane-based chemotherapy for the neoadjuvant treatment of patients with HER2-positive localized breast cancer (BERENICE): A phase II, open-label, multicenter, multinational cardiac safety study. Ann Oncol 29: 646-653, 2018.

14. Gleeson JP, Keegan NM and Morris PG: Adding pertuzumab to trastuzumab and taxanes in HER 2 positive breast cancer. Expert Opin Biol Ther 18: 251-262, 2018.

15. Nami B, Maadi $\mathrm{H}$ and Wang Z: Mechanisms underlying the action and synergism of trastuzumab and pertuzumab in targeting HER2-positive breast cancer. Cancers (Basel) 10: pii: E342, 2018

16. Chang JC, Wooten EC, Tsimelzon A, Hilsenbeck SG, Gutierrez MC, Elledge R, Mohsin S, Osborne CK, Chamness GC, Allred DC and O'Connell P: Gene expression profiling for the prediction of therapeutic response to docetaxel in patients with breast cancer. Lancet 362: 362-369, 2003.

17. Burcombe RJ, Makris A, Richman PI, Daley FM, Noble S, Pittam M, Wright D, Allen SA, Dove J and Wilson GD: Evaluation of ER, PgR, HER-2 and Ki-67 as predictors of response to neoadjuvant anthracycline chemotherapy for operable breast cancer. $\mathrm{Br}$ J Cancer 92: 147-155, 2005.

18. Liu YL, Connolly EP and Kalinsky K: Obesity's impact on survival is independent of dose adjustments in neoadjuvant chemotherapy in women with breast cancer. Breast Cancer Res Treat 168: 285, 2018.

19. Liu YL, Saraf A, Catanese B, Lee SM, Zhang Y, Connolly EP and Kalinsky K: Obesity and survival in the neoadjuvant breast cancer setting: Role of tumor subtype in an ethnically diverse population. Breast Cancer Res Treat 167: 277-288, 2018.

20. Karatas F, Erdem GU, Sahin S, Aytekin A, Yuce D, Sever AR, Babacan T, Ates O, Ozisik Y and Altundag K: Obesity is an independent prognostic factor of decreased pathological complete response to neoadjuvant chemotherapy in breast cancer patients. Breast 32: 237-244, 2017.

21. Fontanella C, Lederer B, Gade S, Vanoppen M, Blohmer JU, Costa SD, Denkert C, Eidtmann H, Gerber B, Hanusch C, et al: Impact of body mass index on neoadjuvant treatment outcome: A pooled analysis of eight prospective neoadjuvant breast cancer trials. Breast Cancer Res Treat 150: 127-139, 2015. 